\title{
A new automated smart cart system for modern shopping centres
}

\author{
Habeeb Bello-Salau', Adeiza James Onumanyi ${ }^{2}$, David Michael ${ }^{3}$, Ridwanullahi Isa ${ }^{4}$, Caroline 0. \\ Alenoghena $^{5}$, Henry Ohize ${ }^{6}$ \\ ${ }^{1}$ Department of Computer Engineering, Ahmadu Bello University Zaria, Nigeria \\ 2,3,4,5 Department of Telecommunication Engineering, Federal University of Technology, Minna, Nigeria \\ ${ }^{6}$ Department of Electrical and Electronic Engineering, Federal University of Technology, Minna, Nigeria
}

\begin{tabular}{l} 
Article Info \\
\hline Article history: \\
Received Dec 31, 2020 \\
Revised Mar 25, 2021 \\
Accepted Jun 14, 202 \\
\hline Keywords: \\
Automated shopping \\
Automatic cart \\
Database \\
Raspberry Pi \\
RFID \\
Supermarket
\end{tabular}

Supermarket

\begin{abstract}
Modern shopping centres are undoubtedly a beehive of intense shopping activities. However, customers are often plagued by salient challenges, which may include fatigue derived from pushing trolleys around the mall and prolonged sorting of bills by the cashier. These shopping challenges could be daunting for the elderly, disabled, pregnant and nursing mothers. In this paper, we addressed these shopping challenges by developing an autonomous shopping cart with the following characteristics; (1) it follows the customer's movement relieving the need to push a cart, (2) it bills automatically all stock placed in the cart, (3) it prompts the customer to make payment and updates each stock via a local database. Our design adopts a Raspberry Pi, a camera and a few direct current motors programmed to achieve autonomy. We used an open-source cross platform software called XAMPP to create the database and used RFID tags to bill the items placed in the cart automatically. The system updates payments and communicates these transactions to a local database via nRF24 wireless transceivers. The experimental tests conducted demonstrate that our system successfully followed customers accurately within the mall. We consider our design a major contribution to the vision of automated shopping systems for the near future.
\end{abstract}

This is an open access article under the CC BY-SA license.

\section{Corresponding Author:}

Habeeb Bello-Salau

Department of Computer Engineering

Ahmadu Bello University Zaria, Nigeria

Email: bellosalau@abu.edu.ng

\section{INTRODUCTION}

In modern shopping centres (malls and super markets), shopping carts are often seen as plastic or metal basket-like frames on wheels pushed by customers to carry items or products around the centre [1], [2]. However, shopping via traditional shopping carts in modern centres may pose several challenges to customers including the burden of pushing heavy carts by the elderly, physically challenged, pregnant and nursing mothers. Other salient challenges may include delay at long queues, prolonged sorting and billing details at the till. Furthermore, mall managers often need to track manually each stock available and sold in the large centre, which can be burdensome. These challenges require innovative solutions, which served to motivate the ideas demonstrated in this paper.

Several authors have proposed different approaches to address the above challenges in the literature. However, these efforts focused more on autonomous mobility of the shopping cart based on the line-following approach [3]-[7]. Image processing has found application in many fields among which include [8], [9]. Its used for autonomous mobility of shopping cart based on some image colours for tracking object 
as well as some android application-controlled approaches were presented in [10]-[14]. While, other efforts addressed automated billing system issues and developed predictive algorithms to suggests items to customers [15]-[17]. A multifunctional automated trolley system based on an android application controlled via a smart phone fixed to a trolley was presented in [2]. Their system achieved good performance. However, it required a predefined black marked line in order to guide the cart's movement. These predefined markers typically constrain the customer's movement thus limiting the flexibility of their design. Similarly, authors in [3] used a line-following robot to drive a shopping cart. They deployed an ultrasonic and line sensor to track the customer's movement and an android application to guide the customer to where goods were located in the mall. They used also an RFID localization scheme and an android application to track the trolley's location. Nevertheless, the line following approach in [3] limits the flexibility of their design similar to [2].

Authors in [4]-[6] used ultrasonic sensors to detect obstacles and to track customers. They used Arduino integrated development environment (IDE) to drive a system of motors to provide autonomous mobility to the cart. Their use of ultrasonic sensors may be similar to other use-cases found in [18], [19]. However, their designs in [4]-[6] are typically unable to differentiate actual users from other stray carts that infiltrate the range of the ultrasonic sensor. A mobile following robot capable of avoiding obstacles, while tracking user was developed in [7]. By using preprogramed images of different objects, authors demonstrated that their system could successfully track customers. A similar concept was presented in [20] that could navigate and avoid obstacles. Authors used this concept to develop autonomous shopping carts. Authors in [21] developed an algorithm to search and map the position of a cart user. They used laser range sensors to estimate a user's position and distance, a gyro-sensor and a rotary encoder. However, their system's accuracy depends strongly on the mapping algorithm, which can be often slow due to complex computations.

Kim et al. [22] investigated different human perspectives toward the design of a convenient and efficient smart cart system. They used a questionnaire and survey method to investigate the best position of an RFID tag and reader, touch screen, a barcode system and a cart module in the smart cart system. The results obtained showed that most customers prefered the touchscreen to be located at about $250 \mathrm{~mm}$ from the handle of the cart, while the cart handle should be tilted at 45 degrees. A similar feasibility study was conducted in [23] to develop an intelligent cart system for use in airports. Authors used the ant colony clustering (ACC) algorithm to find quasi-optimal positions to assemble the cart. The waiting time and precise route of the carts were determined via simulation and detailed procedures were used to compute a sequence of control commands to move the cart. Authors provided results to show that their approach aligned and arranged scattered carts automatically. Marchetti et al. [15], a data fusion algorithm based on particle filter was developed to combine information from an RGB-D camera and a planer laser range finder to estimate and detect the position of a user. These were incorporated into a proposed autonomous shopping cart to track users. To estimate the user's position, authors in [15] adopted a k-clustering technique. They demonstrated that their system consistently tracked the users. Prerana et al. [16] developed an approach that used an RFID reader, which automatically scanned and billed products dropped in the cart. The system added the cost of an item automatically to a list of billed items once the RFID reader scanned it. The customer would activate a switch button to detect the product code in order to remove items no longer required. The system transfers the final product bill via zigbee to the counter where payment can be made. Despite demonstrating the capability of autonomous billing, the system in [16] fails to incorporate autonomous mobility and automatic payment systems in their model.

The approach in [17] used machine learning technique to enable a robot to learn and modify its knowledge about different customer shopping preferences. The system logged a repository of digital data about the user's cultural preferences into a database. It used this information to profile each user and to predict each user's preference. In their design, an inbuilt camera captures images used to train the system to identify the user's emotional status. The results obtained demonstrated the feasibility of their approach. However, their approach is relatively complex because it requires large processing capabilities, which makes it costly for real time realization and implementation.

Inspired by past efforts, we provide in this paper an encompassing design of an automated shopping cart system that improves flexibility, provides instantaneous billing and automated inventory tracking via a local database. The proposed new automated smart cart system used a simple combination of an ultrasonic sensor, Raspberry Pi, some direct current (dc) motors, a video camera, RFID tags and readers, an nRF24 wireless transceiver, an LCD display, a GSM module and a database. Our design provides a low cost innovative smart shopping cart that tracks accurately the customer, provides automatic billing, and displays each unit cost and the total cost of each item on an LCD screen. Furthermore, our system keeps track of all inventories for shop managers/owners. We improved on the approach proposed in [5], [6] by using ultrasonic sensors instead of the line-following technique. We improved also the billing system over the approach in [16] by providing a notification system along with using a local database to keep track and update of stocks in the mall for the shop owner. In addition, we conducted a detailed analysis to examine the performance of our system and its suitability for commercial production. The remainder of the paper is structured as shown 
in: section 2 presents the research method of the proposed smart innovative shopping cart system. Section 3 presents the experimental results and discussion while section 4 concludes the paper.

\section{RESEARCH METHOD}

We adopted a three-stage methodology in our design namely, (1) to develop the autonomous smart cart system (2) to build the billing system and (3) to design the database modules. A schematic of our design is shown in Figure 1 and we explain each stage as:

\subsection{Autonomous smart cart development}

The autonomous smart cart consists of a reinforced aluminium sheet frame supported on a two wheel and a rear-supporting wheel. It contains a basket-like opening, where customers could drop items while shopping. A central processing unit (Raspberry Pi) coordinates and controls the movement of the cart. To provide autonomous mobility to the cart, we adopted an image-processing algorithm based on the RGB colour-processing model [24]. The algorithm acquires an object frame via a camera installed in the top front of the smart cart system, which makes up the video acquisition unit as shown in Figure 1. Similar to the threshold computation approach presented in [25], the algorithm compares each newly acquired object frame with pre-saved object frames in the system and then computes a threshold pixel level using (1).

$$
D=\sqrt{\left(R_{2}-R_{1}\right)^{2}+\left(G_{2}-G_{1}\right)^{2}+\left(B_{2}-B_{1}\right)^{2}}
$$

where, $R_{2}, \mathrm{G}_{2} \& B_{2}$ are the current object image pixel acquired, while $R_{1}, \mathrm{G}_{1} \& B_{1}$ are the pre-saved background object image pixel. If the computed threshold level is less than 500pixel value, the system assumes that the user is found. Else, if the computed pixel value is higher, then the user is assumed not to be within the range of detection by the smart cart. The system adjusts always the location of the pixels computed after detection to the centre of the camera. It uses this adjustment to direct the cart to go either left or right for the horizontal axis and to go either forward or standby for the vertical axis. This guarantees that the user is continuously tracked.

The system acquires two-dimensional object images $(x, y)$ through the camera to determine continuously the position of the user. We used an object-image window size of 320 by 240 pixels to determine the user's position. Usually, if the computed horizontal axis (x-coordinate) of the image is less than 140 pixels, the cart detects the user to be at the right, but if it is greater than 200 pixels, then the cart detects the user to be at the left side due to a 180-degree image inversion of the camera. Similarly, if the computed vertical axis (y-coordinate) of the object image is greater than 170 pixels, the cart assumes that the user is a little farther away and triggers the motor incorporated in the driver unit to move the cart. Else, if otherwise, the cart remains in the standby mode. We provide a summary of this image-processing algorithm in Algorithm 1. The driver unit comprises of a motor driver integrated circuit (L298N), two 12 volts dc motors and a battery. The output of the video acquisition and processing unit trigger the motor driver via the Raspberry Pi processor. We labelled the two de motors as motor 1 and 2, respectively. Each motor had two terminals labelled as A and B and controlled by two logic states 1 and 0 . The logic 1 signifies a high (on) state, while logic 0 signifies a low (off) state. When motor 1 and 2 received a logic combination of 1 and 0 at terminal A and B respectively, it implies that the cart will move in the forward direction. If any of the terminals is reversed for each motor, then the cart will move either left or right direction. However, if all the terminals are at logic 1 or 0 for both motors, it implies that the cart will be on standby mode, i.e. in a stationary position. We summarize in Table 1 the movement pattern of the proposed cart based on the logic received from the Raspberry $\mathrm{Pi}$. Note in Table 1, that N/A denotes that the coordinate values are not applicable to the movement pattern of the cart.

In the obstacle detection unit, an ultrasonic sensor, which is a low-cost transceiver, was utilized. The ultrasonic sensor has a separation distance of $2 \mathrm{~cm}$ between the transmitter and receiver, which was used to approximate the distance between the transmitted ultrasound signals at $40 \mathrm{~Hz}$ from the cart. This determined the time taken to receive the reflected echo pulse from the obstacle along the path as shown in Figure 2. We used an HCSR04 ultrasonic sensor to measure the distance between ranges of $2-400 \mathrm{~cm}$. If the measured distance by the ultrasonic sensor using (2) is less than the set threshold value $(20 \mathrm{~cm})$, the Raspberry Pi triggers the cart to remain in a standby mode since an obstacle apparently exists in its path. Other details and configuration of the principle of operation of the ultrasonic sensor used for the obstacle detection can be seen in [18]. We used an open source processing software environment to program the Raspberry Pi.

$$
d=\frac{h e \times v}{2}
$$

Bulletin of Electr Eng \& Inf, Vol. 10, No. 4, August 2021 : 2028 - 2036 
where $d$ is the distance computed in $(\mathrm{cm})$, heis the echo pulse width high time, and $v$ is Sound velocity $(\mathrm{m} / \mathrm{s})$. The sound velocity is $340 \mathrm{~m} / \mathrm{s}$ or 29 microseconds per $\mathrm{cm}$.

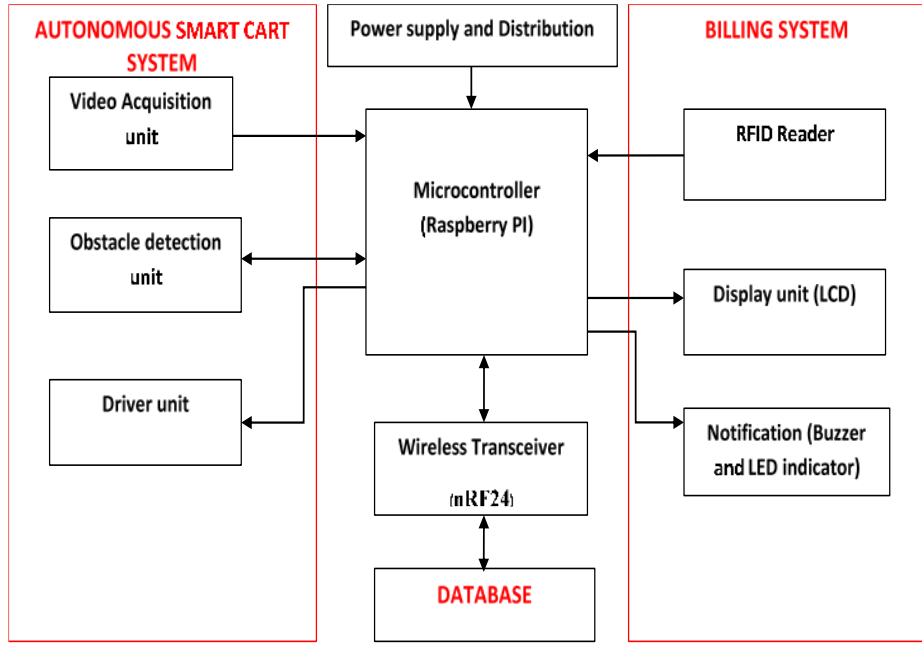

Figure 1. Block diagram of the proposed system architecture and implementation

\section{ALGORITHM 1}

1. Initialise the cart

2. The Microcontroller triggers the video camera.

3. Microcontroller get the background object data from the camera feed.

4. Microcontroller process the background data and presave it.

5. Microcontroller continuously get video frame of the current object.

6. Measure the difference between the current object data and the pre-saved data.

7. Is the difference below the set threshold? If yes,

If No,

a. object found go to step 8

b. Object not found go to step 5 .

8. Compute the horizontal position of the object pixel $(x)$ Else, a. if the $x$-coordinate is less than 140, turn right

$b$. If the $x$-coordinate is greater than 200, turn left.

9. Compute the vertical position of the object pixel (y) a. if the y-coordinate is less than 170, move forward Else

b. Remain in standby mode

10. Return to step 5

Table 1. Proposed smart cart movement pattern

\begin{tabular}{|c|c|c|c|c|}
\hline $\mathrm{x}$-coordinate & $y$-coordinate & Direction & Motor I & Motor 2 \\
\hline N/A & $<170$ & Forward & A: 1 B: 0 & A: 1 B: 0 \\
\hline N/A & $>170$ & Standby & A: 1 B: 1 & A: 1 B: 1 \\
\hline$<140$ & N/A & Turn Right & A: 0 B: 1 & A: 1 B: 0 \\
\hline$>200$ & N/A & Turn Left & A: 1 B: 0 & A: 0 B: 1 \\
\hline
\end{tabular}

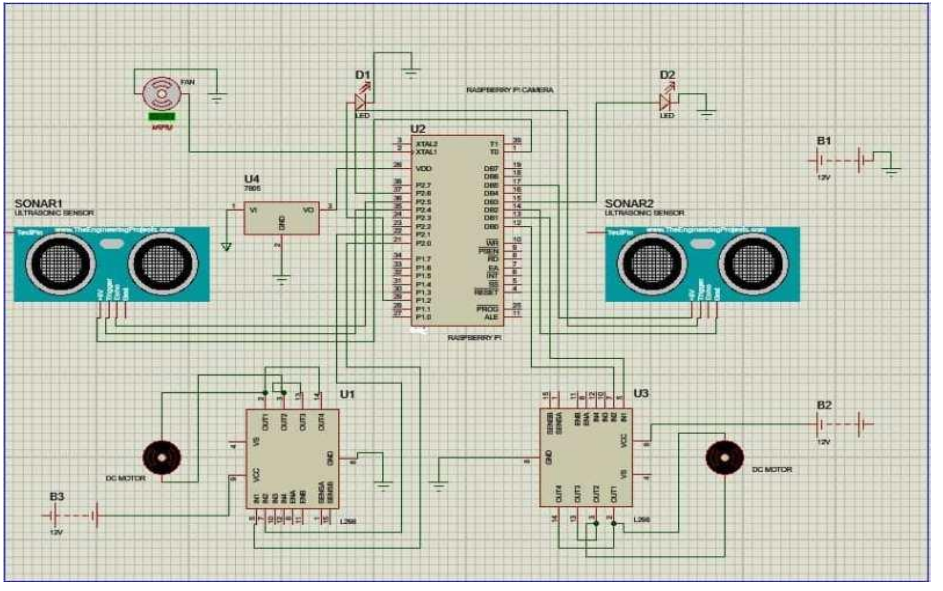

Figure 2. Circuit diagram of the autonomous cart

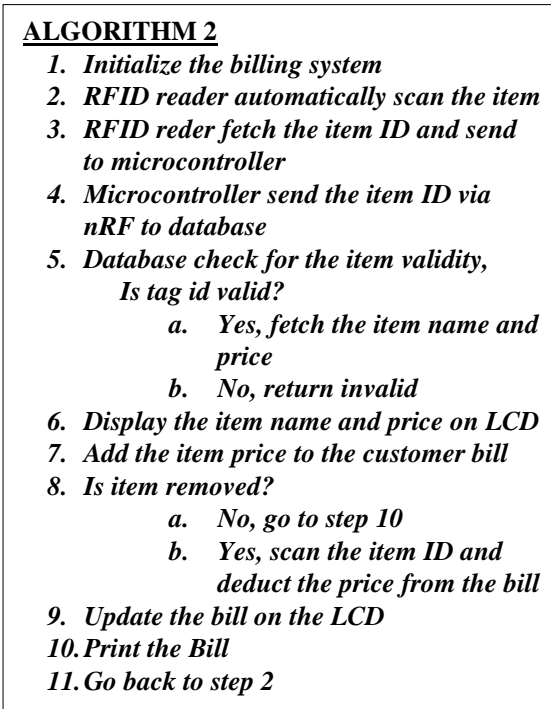

2. $R F I D$ reader automatically scan the item

$D$ reder fetch the

Microcontroller send the item ID via nRF to database

stag id valid?

price

6. Display the item name and price on $L C D$

7. Add the item price to the customer bill

go to step 10

Yes, scan the item ID and

9. Update the bill on the $L C D$

11. Go back to step 2

\subsection{The billing system}

This module comprises of an RFID reader operating at a frequency of $125 \mathrm{kHz}$, incorporated inside the basket-like opening in the smart cart. We tagged each item using a unique ID in all our experiments. This enabled the system to scan automatically each item placed in the cart. Usually, the RFID reader would read these IDs via the serial pin of the Arduino Uno board. The Arduino board controls the billing module, as information received from the transmitter (RFID reader) is sent to the developed database as shown in 2.3. It achieves this via the nRF24 wireless transceiver, interfaced to the Arduino using a serial peripheral interface 
(SPI). We covered the outer part of the smart cart with a radio frequency (RF) shield to prevent the RFID reader from scanning other products outside the cart. The system extracts from a database the name and cost of each item placed in the cart and displays these on an LCD. As the items placed in the cart increases, the cost of each item is added to the cost previously extracted from the database and this is displayed on the LCD. When a user changes his/her mind about an item and removes it from the cart, the RFID scans automatically the item and communicates its ID to the database, which updates automatically the list of intended purchased items and their corresponding cost. We summarize this entire process in Algorithm 2.

\subsection{Database module}

This section describes the local database that contains information about each item stocked in the mall. We created the database using the MySQL and the XAMPP control panel, which is an open source cross platform. We used the MySQL and Apache from the XAMPP control panel in our design. The domain "localhost/phpmyadmin/" was launched via the address bar of a Google Chrome browser. We named the database as "shopping cart and table" and displayed this on the phpmyadmin interface, which indicates that the database was created successfully. The phpmyadmin interface launches the created "shopping cart and table" database. The "Database Shopping Cart" was assigned. Five different fields namely; (1) serial number (s/n), (2) tag ID, (3) item name, (4) item price, and (5) amount in stock. The data type for each field was assigned and the data format type called "integer" was assigned to $\mathrm{S} / \mathrm{N}$ and to amount in stock, while the variable character is assigned to all other fields. Further details concerning the creation of the database with other necessary fields using the XAMPP software can be found in [26]. The database was interfaced with an ethernet shield and an Arduino Uno board, which retrieves from the billing module any necessary information requested by the item ID as shown in section 2.2. The retrieved information from the local database is sent to the billing module via the ethernet shield and the nRF transceiver. The overall system architecture comprising of all the three modules is summarised in Figure 3.

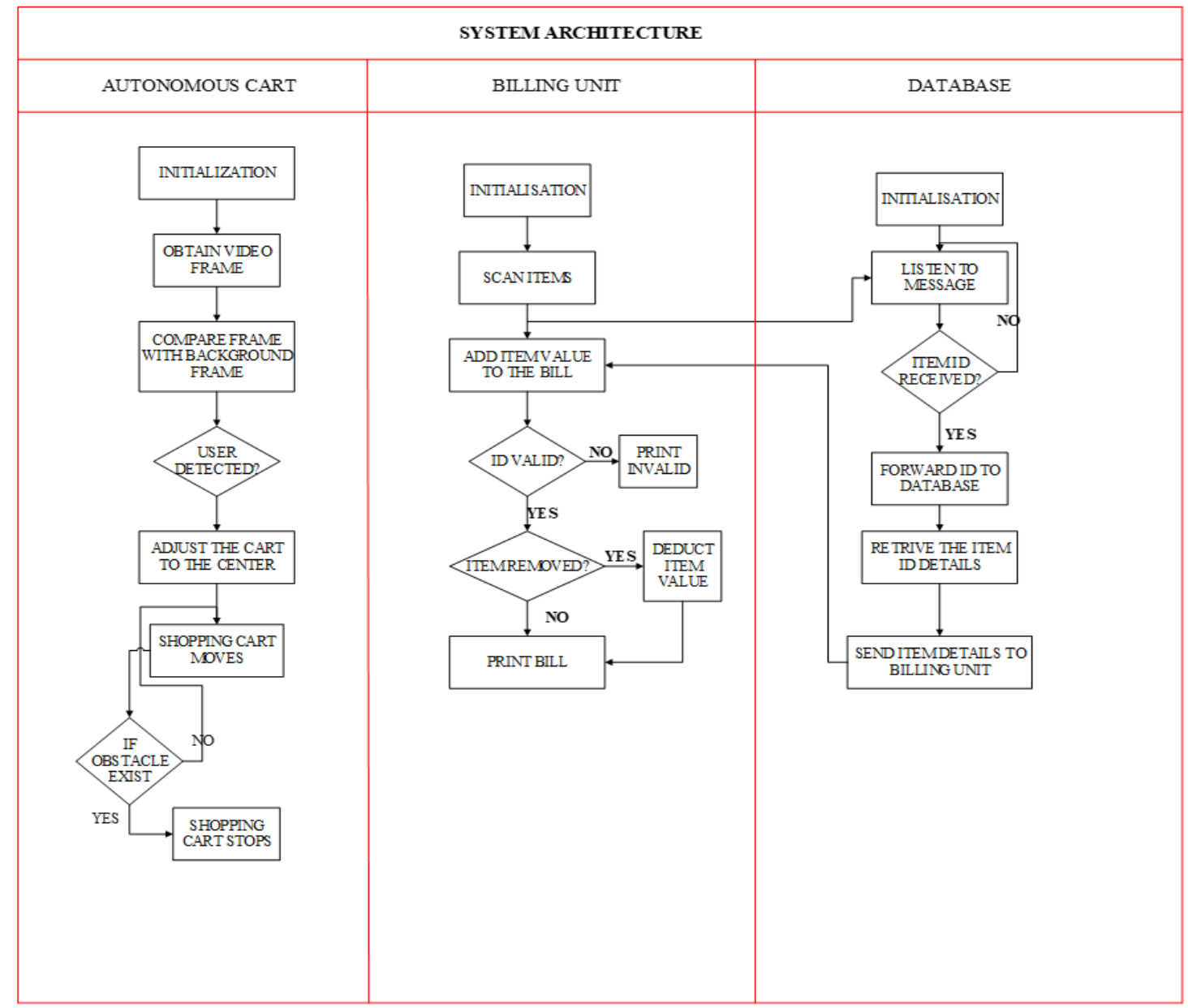

Figure 3. Overall system architecture methodology flowchart 


\section{RESULTS AND DISCUSSION}

We tested the new smart cart system in two stages. First, we tested separately the individual modules of the proposed system viz the autonomous module, the billing and database module. Secondly, we tested the communication system between all the three modules as well as the entire system. In testing the autonomous module of the proposed system, the camera feed rate was set to 30 frames per seconds, while the best orientation of the camera that gives better result was investigated as summarized in Table 2. Observe that an average response time of $33 \mathrm{~ms}$ was required to trigger the autonomous tracking of the user. We designed the billing system to scan an item at a time, particularly as each item was dropped in the cart. The system had a delay of $500 \mathrm{~ms}$ after scanning automatically the item placed inside the cart. This delay was necessary in our design to enable the system to communicate with the local database in order to fetch the cost of each item, compute and sum the total bill of items in the cart. This was initialized by the pre-saved colour of an object that will usually be attached to the user's body. We measured this response time only when the user is within the range of operation of the proposed system, which is in the range of 0 to $475 \mathrm{~cm}$. The system becomes non-responsive at ranges greater than $475 \mathrm{~cm}$ as shown in Table 2. The system remained in a standby mode when the user was positioned within a range of $0-50 \mathrm{~cm}$. In this case, it did not matter whether the user was positioned directly in front of the cart or on its sides. In addition, the system performed well in terms of successfully tracking the user, especially when the user was positioned directly in front of the cart. However, it performed poorly when the user was positioned at the bottom plane as shown in Table 2. Generally, an average-response scan time of about $300 \mathrm{~ms}$ after 60 trials was recorded for each item as shown in Table 3), with a corresponding bill computation time of less than $850 \mathrm{~ms}$ at varying distances between 1 to $5 \mathrm{~cm}$. Nevertheless, the system may remain non-responsive and thus continue to scan indefinitely when the scanning distance is greater than $5 \mathrm{~cm}$ as shown in Table 3 . The screen shot of the created database structure and its fields are shown in Figure 4. We provide also in Figure 5 an image of the physical prototype of the cart system.

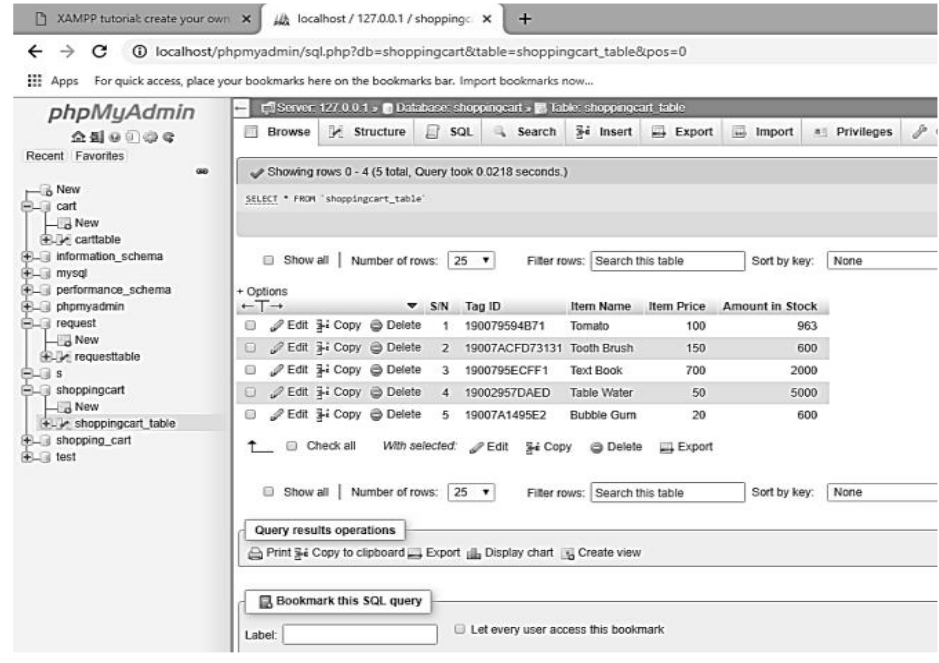

Figure 4. Screenshot of the created database

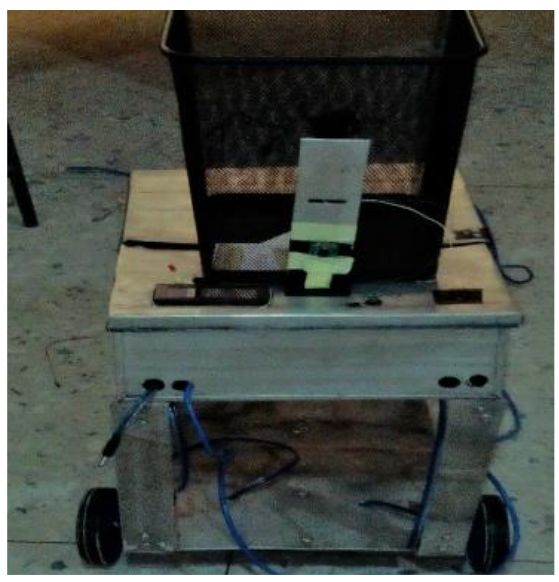

Figure 5. Prototype of the prposed innovative shopping cart system

Table 2. Performance response time and tracking by the proposed system

\begin{tabular}{ccccc}
\hline Position & Range $(\mathrm{cm})$ & Response time $(\mathrm{ms})$ & Action & Performance \\
\hline Sides (right/left) & $0-50$ & 33 & Standby & Fair \\
& $50-475$ & 33 & Left/right/forward & \\
Top (center) & $>500$ & No response & & \\
& $0-50$ & 33 & Stand by & Very good \\
& $50-450$ & 33 & Left/right/forward & \\
Bottom plane & $>485$ & No response & & Poor \\
& $0-50$ & 33 & Standby & \\
& $50-185$ & 33 & Left/right/forward & \\
\hline
\end{tabular}


Table 3. Average scan duration and response time of billing module

\begin{tabular}{ccc}
\hline Scan distance $(\mathrm{cm})$ & Acerage scan duration & Average response time (bill computation) \\
\hline 1 & $<300 \mathrm{~ms}$ & $<850 \mathrm{~ms}$ \\
2 & $<300 \mathrm{~ms}$ & $<850 \mathrm{~ms}$ \\
3 & $<300 \mathrm{~ms}$ & $<850 \mathrm{~ms}$ \\
4 & $<300 \mathrm{~ms}$ & $<850 \mathrm{~ms}$ \\
5 & $<300 \mathrm{~ms}$ & $<850 \mathrm{~ms}$ \\
6 & Infinite & No response \\
\hline
\end{tabular}

\section{CONCLUSION}

This paper has presented the design and prototyping of an innovative smart shopping cart system. The autonomous capability of the system was achieved using pre-saved object images that were processed and used to trigger the mobility of the cart. A Raspberry Pi processor was used to coordinate the cart's movement. In addition, a local database was created using XAMPP software, which contained the stocked items and their corresponding ID, price and the number of stocks. The database keeps track of available stocks in the mall. Furthermore, the billing module of our system communicates via the nRF24 wireless transceiver to fetch information required from the database. This was achieved by scanning automatically the item's ID as they were dropped in the cart. Our test results showed that the cart system tracks and follows the user successfully and automatically particularly when it was triggered by the pre-saved colour image of an object placed on the user's body. Our system also billed items automatically that were dropped in the cart. It similarly updates successfully the stock on the database. Future works will consider how to integrate in our design an online payment unit in addition to providing automatic battery charging and cart parking capability, this will further improve the efficacy and performance of the system.

\section{REFERENCES}

[1] B. G. Mchale, D. C. Winkle, M. D. Atchley, S. Chakrobartty, and D. R. High, "Shopping facility assistance system and method having a motorized transport unit that selectively leads or follows a user within a shopping facility," in Google Patents, 2019.

[2] L. Dehigaspege, M. Liyanage, N. Liyanage, M. Marzook, and L. Dhammearatchi, "Follow Me Multifunctional Automated Trolley," International Journal of Engineering Research \& Technology (IJERT), vol. 6, no. 7, pp. 8490, 2017.

[3] Y. L. Ng, C. S. Lim, K. A. Danapalasingam, M. L. P. Tan, and C. W. Tan, "Automatic human guided shopping trolley with smart shopping system," Jurnal Teknologi, vol. 73, no. 3, 2015, doi: 10.11113/jt.v73.4246.

[4] E. D. Suryanto, H. Siagian, D. Perangin-Angin, R. Sashanti, and S. Yogen, "Design of automatic mobile trolley using ultrasonic sensors," in Journal of Physics: Conference Series, 2018, vol. 1007, no. 1, p. 012058, doi: 10.1088/1742-6596/1007/1/012058.

[5] H. Lim, Y. Hwang, and J. L. K. Kim, "Object-tracking robot using ultrasonic sensor and servo motor," in The Fourth International Conference on Smart IT Aplications, 2012, vol. 1.

[6] C. Nuñez, A. García, R. Onetto, D. Alonzo, and S. Tosunoglu, "Electronic Luggage Follower," in Florida Conference on Recent Advances in Robotics, FCRAR, 2010, pp. 20-21.

[7] T. Subramanian, R. Madbhavi, S. Potadar, S. J. D'souza, S. Gangadharan, "Object Follower and Barrier Escaping Robot Using Image Processing," International Journal of Innovative Research in Science, Engineering and Technology, pp. 2319-8753, 2015.

[8] H H. Bello-Salau, A. M. Aibinu, E. N. Onwuka, J. J. Dukiya and A. J. Onumanyi, "Image processing techniques for automated road defect detection: A survey," 2014 11th International Conference on Electronics, Computer and Computation (ICECCO), 2014, pp. 1-4, doi: 10.1109/ICECCO.2014.6997556.

[9] H H. Bello-Salau, A. J. Onumanyi, A. T. Salawudeen, M. B. Mu'azu and A. M. Oyinbo, "An Examination of Different Vision based Approaches for Road Anomaly Detection," 2019 2nd International Conference of the IEEE Nigeria Computer Chapter (NigeriaComputConf), 2019, pp. 1-6, doi: 10.1109/NigeriaComputConf45974.2019.8949646.

[10] M. M. Islam, A. Lam, H. Fukuda, Y. Kobayashi, and Y. Kuno, "A person-following shopping support robot based on human pose skeleton data and lidar sensor," in International Conference on Intelligent Computing, 2019, pp. 919:, doi: 10.1007/978-3-030-26766-7_2.

[11] N. A. Rawashdeh, R. M. Haddad, O. A. Jadallah and A. E. To'ma, "A person-following robotic cart controlled via a smartphone application: design and evaluation," 2017 International Conference on Research and Education in Mechatronics (REM), 2017, pp. 1-5, doi: 10.1109/REM.2017.8075245.

[12] A. A. Gunawan et al., "Development of Smart Trolley System Based on Android Smartphone Sensors," Procedia Computer Science, vol. 157, pp. 629-637, 2019, doi: 10.1016/j.procs.2019.08.225.

[13] R. Dhianeswar, M. Gowtham, and S. Sumathi, "Smart Trolley with Automatic Master Follower and Billing System," in International conference on Computer Networks, Big data and IoT, 2018, pp. 778-791, doi: 10.1007/978-3-030-24643-3_92. 
[14] D. Lestari, S. Sendari, S. D. Sanjaya, A. N. Handayani, H. Lin and A. B. Fahmi, "Locking Object Position using Camshift Algorithm for Automatic Trolley," 2019 International Conference on Electrical, Electronics and Information Engineering (ICEEIE), 2019, pp. 90-95, doi: 10.1109/ICEEIE47180.2019.8981447.

[15] L. Marchetti, D. Pucci, and P. Morin, "Autonomous shopping cart platform for people with mobility impairments," in Workshop at IROS'12 on Assistance and Service robotics in a human environment., 2012.

[16] T. Prerana, S. Ranjan, D. Kaushik, and Research, "Smart Shopping Cart for Automatic Billing in Supermarket," International Journal of Engineering Development and Research, vol. 5, no. 2, pp. 975-978, 2017.

[17] F. Bertacchini, E. Bilotta, and P. B. Pantano, "Shopping with a robotic companion," Computers in Human Behavior, vol. 77, pp. 382-395, 2017, doi: 10.1016/j.chb.2017.02.064.

[18] H. Bello-Salau, A. Aibinu, E. Onwuka, J. Dukiya, A. Onumanyi, and A. Ighabon, "Development of a laboratory model for automated road defect detection," Journal of Telecommunication, Electronic and Computer Engineering (JTEC), vol. 8, no. 9, pp. 97-101, 2016.

[19] H. Bello-Salau et al., "A new measure for analysing accelerometer data towards developing efficient road defect profiling systems," Journal of Scientific Research \& Reports, vol. 7, no. 2, pp. 108-116, 2015, doi: 10.9734/JSRR/2015/16840.

[20] R. C. Kumar et al., "Obstacle avoiding robot-a promising one," International Journal of Advanced Research in Electrical, Electronics and Instrumentation Engineering, vol. 2, no. 4, pp. 1430-1434, 2013.

[21] T. Onozato, H. Tamura, Y. Kambayashi, and S. Katayama, "A Control System for the Robot Shopping Cart," in 2010 IRAST International Congress on Computer Applications and Computational Science (CACS 2010), 2010, pp. 907-910, doi: 10.1007/978-3-642-23851-2_29.

[22] M. K. Kim, E. Y. Lee, and Y. M. Chang, "A study on the human factors for a smart cart system," Procedia Manufacturing, vol. 3, pp. 566-573, 2015, doi: 10.1016/j.promfg.2015.07.267.

[23] Y. Kambayashi, H. Yamachi, M. J. C. i. I. S. Takimoto, and M. Engineering, "Feasibility Studies of the Intelligent Cart System: Toward a Practical System," Communications in Information Science and Management Engineering, vol. 2, no. 6, pp. 1-8, 2012.

[24] M. Munaro, F. Basso and E. Menegatti, "Tracking people within groups with RGB-D data," 2012 IEEE/RSJ International Conference on Intelligent Robots and Systems, 2012, pp. 2101-2107, doi: 10.1109/IROS.2012.6385772.

[25] H. Bello-Salau, A. Onumanyi, B. Sadiq, H. Ohize, A. Salawudeen, and A. Aibinu, "An Adaptive wavelet transformation filtering algorithm for improving road anomaly detection and characterization in vehicular technology," International Journal of Electrical \& Computer Engineering (IJECE), vol. 9, no. 5, 2019, doi: 10.11591/ijece.v9i5.pp3664-3670.

[26] H. Bello-Salau, A. Aibinu, A. Onumanyi, S. Ahunsi, E. Onwuka, and J. Dukiya, "Development of a road surface condition monitoring and database system," in 2nd International Conference on Information and Communication Technology and Its Applications (ICTA 2018), Federal University of Technology, Minna, Nigeria, 2018, p. 6.

\section{BIOGRAPHIES OF AUTHORS}

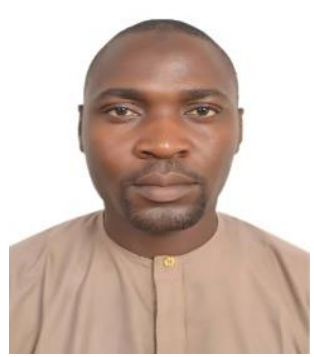

Habeeb Bello Salau (Member, IEEE) received the B.Tech. degree in Electronic and Electrica Engineering from Ladoke Akintola University of Technology, Ogbomoso, Nigeria, in 2009, the M.Sc. degree in Communication Engineering from International Islamic University Malaysia, Kuala-Lumpur, in 2012, and the Ph.D. degree in Communication Engineering from the Federal University of Technology Minna, Nigeria, in 2017. He is currently with the Department of Computer Engineering, Ahmadu Bello University, Zaria, Nigeria. He has authored and coauthored several research articles in peer reviewed journals and in different indexed conferences. He has served as reviewer for different high impact journals and serve as member of the organizing committee of several high impact conferences including IEEE. His research interests include digital signal and image processing, vehicle ad-hoc networks, artificial intelligence, cognitive radio, and wireless sensor networks.

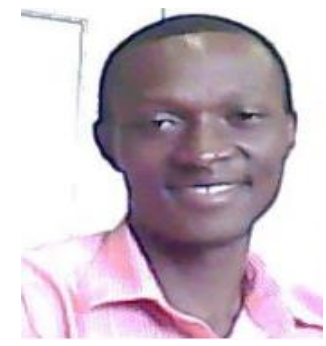

Adeiza James Onumanyi (Member, IEEE) received the B.Eng. degree in electrical and electronics engineering from Abubakar Tafawa Balewa University, Bauchi, Nigeria, in 2005, and the M.Eng. and Ph.D. degrees in communication engineering from the Federal University of Technology (FUT) Minna, Minna, Nigeria, in 2010 and 2014, respectively. He was a Postdoctoral Research Fellow with the University of Pretoria, South Africa, from 2018 to 2019. He is currently a Lecturer with the Department of Telecommunication Engineering, FUT Minna. He has published several research articles in different peer reviewed journals, and in different IEEE flagship conferences. He has won several grants at FUT Minna, served on several organizing committees for different conferences, including the IEEE conferences, reviewed several articles for high impact journals. He has participated in different technical workshops. His research interests include spectrum sensing in cognitive radio, wireless sensor networks, radar systems, image processing, cyber physical systems, and low powered wireless area networks 

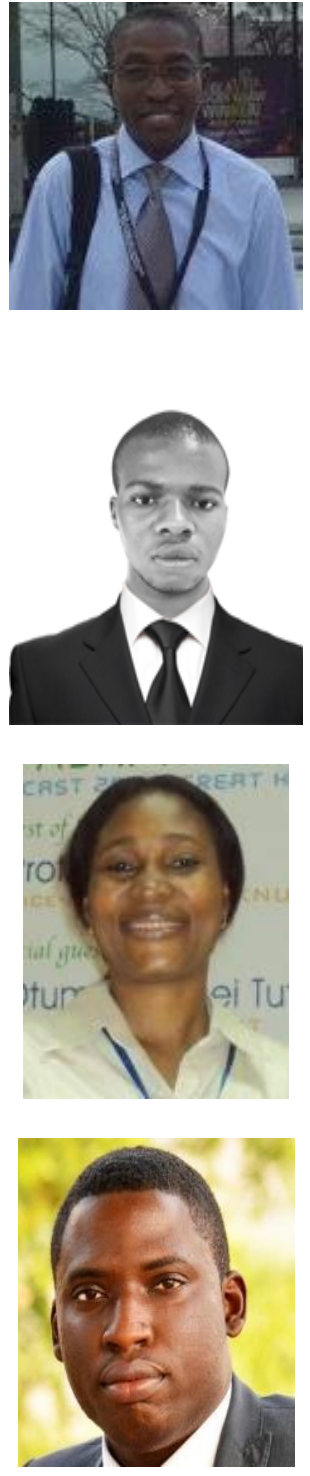

Michael David received his Bachelor of Engineering degree in Electrical and Computer Engineering and Master of Engineering degree in Telecommunication Engineering from Federal University of Technology, Minna, Nigeria in 2004 and 2010, respectively. The Ph.D. degree in Electrical Engineering was conferred on him by Universiti Teknologi Malaysia (UTM), Skudai Johor, Malaysia in 2016 for his work on visible absorption based ozone sensors. He is currently a Lecturer with Federal University of Technology Minna, Nigeria. His research focuses on absorption spectroscopy for gaseous ozone concentration measurement. $\mathrm{He}$ is a registered Engineer with the Council for the regulation of Engineering in Nigeria (COREN). He is also a Certified Fiber Optic Technologist (CFOT) and a member of Fiber Optic Association, inc. USA.

Ridwan Isa Obtained his Bachelor of Engineering degree in Telecommunication Engineering from the School of Electrical Engineering and Technology, Federal University of Technology Minna in 2018. He is currently an MSc student at Air Force Institute of Technology, Kaduna, Nigeria. His current research focus include improvement of drone communication, avionics and embedded system.

Caroline O. Alenoghena holds a $\mathrm{PhD}$ in Telecommunications Engineering, and a Masters degree in Electronics Telecommunications. She is a member of the Institute of Electrical and Electronic Engineers MIEEE, Nigerian Society of Engineers (NSE), and Association of Professional Women Engineers of Nigeria APWEN. She is a registered practicing Engineer with the Council for the Regulation of Engineering in Nigeria. Her research interests include intelligent systems and networks.

Henry Ohize earned a Ph.D. in 2017 from the University of Cape Town, South Africa, a master's degree in Telecommunication Engineering from Federal, University of Technology Minna, Nigeria in 2010 and a Bachelor's degree in Electrical and Electronics Engineering, from the Abubarkar Tafawa Balewa University Bauchi, Nigeria in 2005. He is currently a senior lecturer at the Federal University of Technology, Minna. His research interests include, the full realization and implementation of 5G technology: Cognitive Radio, Wireless Sensor Networks, MIMO, mmWave and 5G application areas. 